Int. J. Speleol. 10 (1978), pp. $381-386$

\title{
Bragasellus escolai n.sp., Crustacea Isopoda Asellota cavernicole d'Espagne
}

\author{
par \\ J.P. HENRY et G. MAGNIEZ*
}

Bragasellus escolai n.sp., cavernicolous Crustacea Isopoda Asellota from Spain:

SUMMARY

Description of a new, unpigmented and anophtalmous species of Bragasellus collected in Calderón Cave, Vellila de Carrión, Palencia Province, Northern Spain (hydrographic basin of Douro River).

ORIGINE: Ce nouvel Asellide a été capturé dans les eaux de la Cueva Caldéron à Velilla de Carrión (93 km au NNW de Palencia), province de Palencia, Espagne septentrionale, au mois d'août 1971 (S.A.S. leg.).

Il nous a été transmis par notre collègue $\mathrm{O}$. Escolà de Barcelone, à qui nous le dédions et que nous remercions très vivement pour sa confiance.

La nouvelle espèce appartient sans ambiguité au genre Bragasellus Henry et Magniez, 1970, Asellides endémiques de la péninsule ibérique dont 8 espèces étaient précédemment décrites (Henry et Magniez, 1977).

LOT CAPTURÉ: 38 individus adultes dont 6 of et 32 q. Taille des $\delta: 4,4$ à 5,3 $\mathrm{mm}$. Taille des $q: 4,9$ à $6,8 \mathrm{~mm}$. Tous sont spécifiquement identiques. Un $\delta$ de $5,3 \mathrm{~mm}$ et une $q$ de $6,5 \mathrm{~mm}$ ont été disséqués pour l'étude des appendices. Les $q$ possèdent des oostégites réduits en forme de lamelles allongées rejoignant presque leur symétrique sur la ligne médiosternale du corps (fig. 9). Elles se trouvent en intermue de repos génital et montrent des ovocytes en voie de croissance dans leurs ovaires. L'importance de leur nombre, la similitude de leur état génital et la date de capture nous incitent à penser que la reproduction de l'espèce est saisonnière dans cette station (ponte au printemps).

* Laboratoire de Biologie Animale et Générale, Université de Dijon, 6, bd. Gabriel, 21100 Dijon, France 


\section{DESCRIPTION:}

ASPECT GÉNÉRAL: Asellide au corps grêle (coefficient d'allongement légèrement supérieur à 5 chez le of́); la $q$ est moins longiligne. Tégument mince et translucide. Chétotaxie générale assez fournie avec de longues soies lisses (jusqu'à $150 \mu$ ) sur les marges latérales des péréionites et du pléotelson. Céphalon subtrapézoïdal, un peu plus large que long, lobes postmandibulaires nettement marqués, yeux nuls.

Marges des péréiopodes I à VII régulières et non échancrées; coxopodites des péréiopodes réduits à un anneau chitineux non apparent en vue tergale (fig. 1). Pléonites I et II libres, bien dégagés, formant un pédoncule au pléotelson (fig. $8)$; celui-ci plus long que large $(C=1,20)$, régulièrement ovalaire, à pointe caudale très atténuée.

Largeurs respectives de la tête, des 7 péréionites libres, des pléonites I et II et du pléotelson chez le ớ de 5,3 mm: 0,83 - 1 - 1,03 - 1,04-1,04 - 1,03 - 1,04 -1,03 $-0,62-0,62-0,98$. Intestin postérieur complètement rempli de limon beige. Une paire de papilles génitales subcylindriques de $250 \mu$ de long et 50-60 $\mu$ de diamètre insérées sur la marge caudale du dernier sternite thoracique du $\delta$.

APPENDICES CÉPHALIQUES: Antennules nettement plus courtes que la hampe de l'antenne; hampe de 4 articles et fouet de 6 articles dont les 5 distaux portent chacun une lame olfactive hyaline de $65 \mu$ de long dans les 2 sexes. Antennes relativement longues ( $5 \mathrm{~mm}$ pour une o de $6,5 \mathrm{~mm}$ ); hampe de 6 articles et fouet grêle et fragile d'une cinquantaine d'articles. Paragnathes formant deux grands lobes subovalaires à marge distale interne portant une brosse de fines soies.

Mandibules très redressées; palpe triarticulé relativement court; processus inciseur à 4 dents; lacinia mobilis puissante (4 dents également), suivie d'une rangée de 9 épines ramifiées (10 épines à droite, dont les 5 distales plus fortes et dentelées); lobe molaire robuste, allongé, garni distalement d'une brosse de courtes soies.

Maxillules typiques: lobe externe avec une douzaine de fortes épines dentelées distales ( 30 à $60 \mu$ de long) et une forte soie lisse sur la face médiane sternale; lobe interne avec 5 fortes épines rameuses distales ( 50 à $70 \mu$ de long). Maxilles trilobées normales: lobe externe avec une rangée distale de 17 lames falcifor-

Fig. 1 à 7 . 1 - Région pleurale gauche des péréionites II et III, face sternale, du 8 de 5,3 mm; e = $200 \mu$

2 - Péréiopode I droit du même individu; $\mathrm{e}=100 \mu$

3 - Péréiopode I droit de la o de $6,5 \mathrm{~mm} ; \mathrm{e}=200 \mu$

4 - Propodite et dactylopodite du péréiopode VII gauche du of de $5,3 \mathrm{~mm}$; e $=100 \mu$.

5 - Pléopode III gauche du même individu; $\mathrm{e}=200 \mu$

6 - Pléopode IV gauche du même individu; $\mathrm{e}=200 \mu$

7 - Pléopode V gauche du même individu; $\mathrm{e}=200 \mu$ 


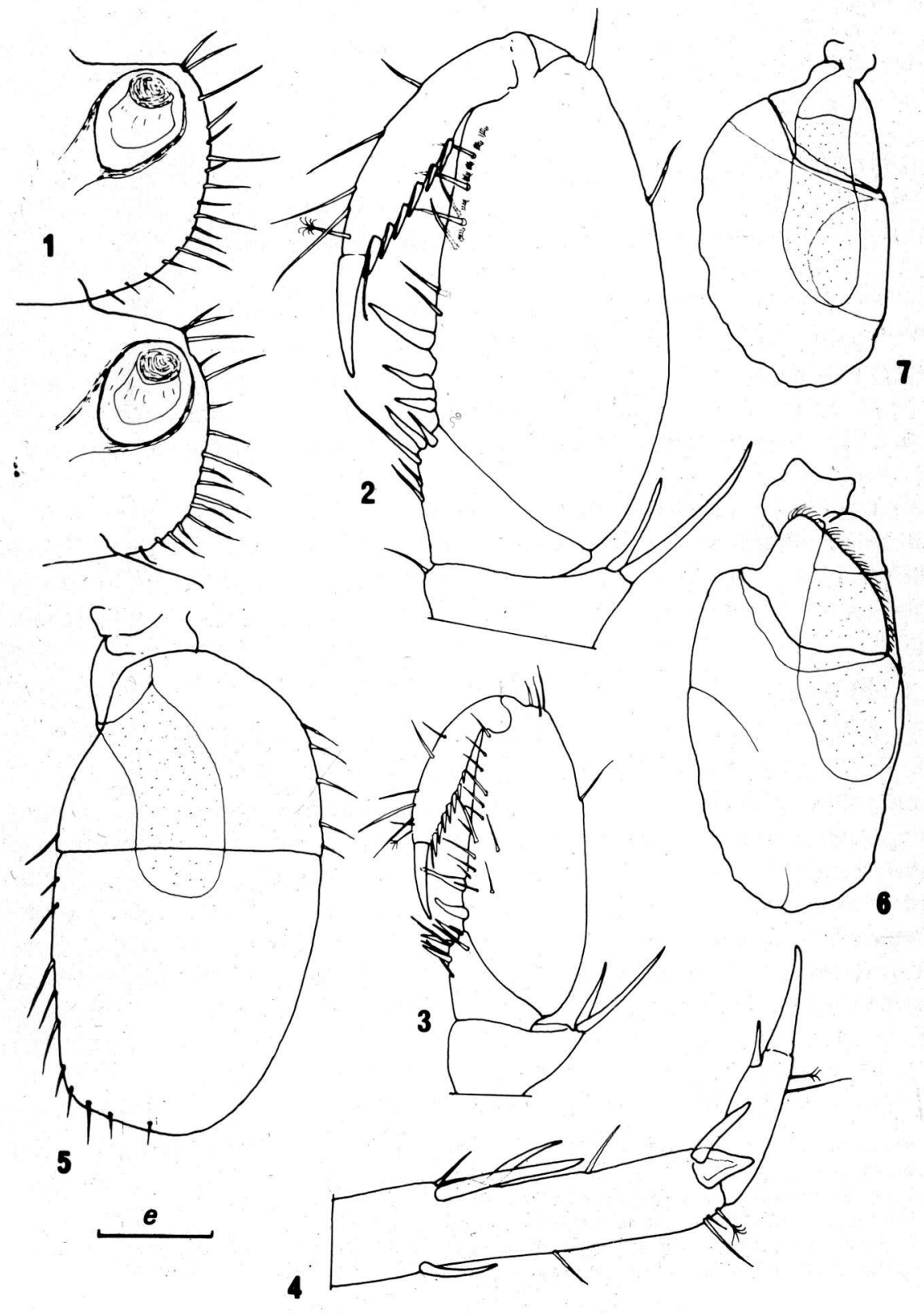


mes pectinées sur la large interne, décroissant de 150 à $40 \mu$ vers l'intérieur; lobe médian avec une rangée identique de 11 tiges (130 à $50 \mu)$; lobe interne avec une double crête, portant, d'une part une vingtaine de tiges dont 9 longues et rameuses $(35-40 \mu)$ et les autres plus courtes $(25-30 \mu)$, intercalées avec les précédentes, incluses dans une plage de fines soies, et d'autre part un peigne interne d'une vingtaine de fines et longues tiges $(60-70 \mu)$.

MAXILLIPÈDES: Typiques d'Asellides, avec un grand épipodite lamelleux, subquadrangulaire muni de 8-14 courtes soies marginales lisses; palpe à 5 articles dont le méropodite très dilaté $(1-20-15-20-25$ soies lisses environ sur les articles 1 à 5); endite avec une forte armature distale et tergale de tiges falciformes rameuses; rétinacle de 5-6 crochets; angle proximal interne du coxopodite avec une petite protubérance charnue chez la $\$$.

PÉRÉIOPODES: Longueurs absolues chez le ơ de 5,3 mm: 1,54 - 1,90 - 2,05 $-2,05-2,28-2,56$ et $2,73 \mathrm{~mm}$; longueurs relatives à P2: $81-100-107-107-119$ - 134 - 142 . Péréiopode I ó (fig.2) à propodite largement renflé, son bord sternal avec 3 phanères ensiformes sur le tiers proximal ainsi que quelques tiges submarginales et écailles denticulées sur les tiers distaux. Dactylopodite avec une série de 6 épines sternales. Propodite I de la $q$ avec seulement 2 phanères ensiformes sternaux mais dactylopodite avec 7 épines sternales. (fig.3). Dactylopodites des péréiopodes II à VII avec une seule épine sternale allongée. (fig.4).

PLÉOPODES I ớ: Typiques du genre: protopodites non soudés entre eux, avec un rétinacle symétrique à 3 crochets; exopodite subovalaire allongé avec une vingtaine de tiges marginales lisses distales et externes (fig. 10).

PLÉOPODES II q: Protopodite environ 1,7 fois plus long que large, quadrangulaire avec exopodite inséré sur la marge distale et endopodite à l'angle distal interne. Exopodite biarticulé avec article proximal plus large que le distal, celui-ci semi-ovalaire avec 9-11 tiges marginales, dont les 5 distales internes serrées, plus longues et légèrement plumeuses (fig.11). Endopodite comportant une courte région proximale dotée d'une apophyse externe massive et séparée du corps vésiculeux de l'organe par une constriction. Distalement on trouve une épaisse protubérance tergale et une longue canule grêle et sternale, légèrement arquée, qui montre un sillon longitudinal hélicoïdal (fig.13). Ori-

Fig. 8 à 14.8 - Péréionite VII et pléon, face tergale, du ${ }^{\prime}$ de $5,3 \mathrm{~mm}$; e $=380 \mu$.

9 - Péréionite III, face sternale, de la $q$ de $6,5, \mathrm{~mm}$, montrant les coxopodites munis d'oostégites non fonctionnels; $\mathrm{e}=400 \mu$.

10- Pléopode I gauche du ơ de 5,3 mm; e $=110 \mu$.

11- Pléopode II droit du même individu; $\mathrm{e}=110 \mu$

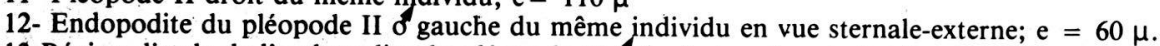

13-Région distale de l'endopodite du pléopode II ơ droit du même individu; e $=40 \mu$.

14-Pléopode II de la $q$ de $6,5 \mathrm{~mm} ; \mathrm{e}=200 \mu$. 


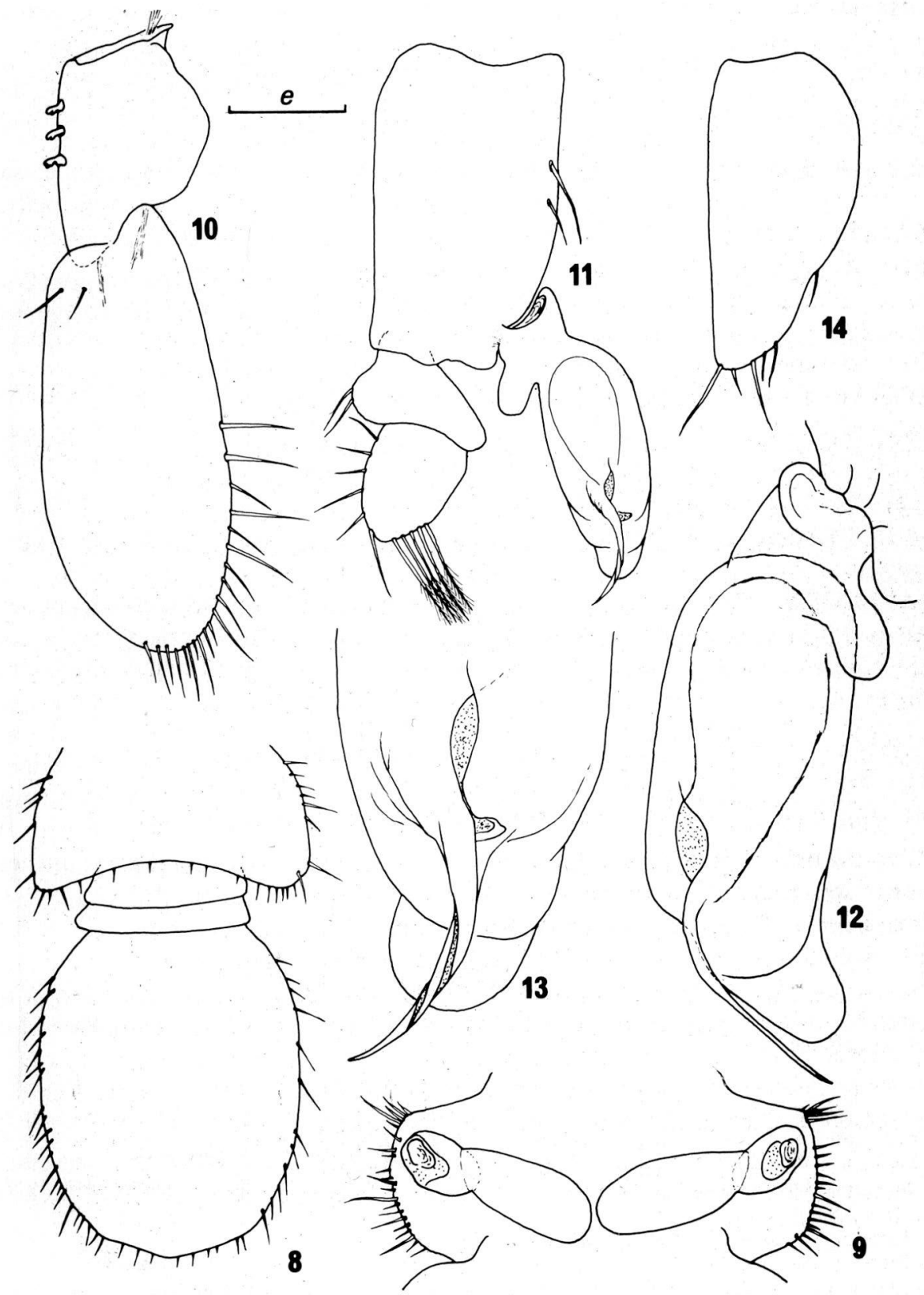


fice afférent de la vésicule interne ovale (fig.12).

PLÉOPODES II ó: Indépendants l'un de l'autre, subtriangulaires, allongés; la soie exopodiale est isolée à la limite d'une encoche de la marge externe; de plus, 4-5 soies lisses sont implantées distalement (fig.14).

PLÉOPODES III: Exopodite (opercule) biarticulé à suture non oblique; sa marge interne et distale porte une douzaine de tiges lisses. Endopodite petit, charnu et à rôle respiratoire (fig. 5).

PLÉOPODES IV: Exopodite ovalaire trés mince, avec une double suture proximale nette, les autres à peine marquées. Endopodite petit, réguliérement ovalaire, charnu et respiratoire (fig.6).

PLÉOPODES V: Exopodite ovalaire, grand et nu; linea duplex visible, linea articularis peu apparente. Endopodite petit, charnu et respiratoire (fig.7). Les endopodites III, IV et V sont porteurs de plusieurs dizaines d'Acinétiens phorétiques sessiles ovoïdes de $15 \times 30 \mu$ environ.

UROPODES: Grêles et fragiles, ils atteignent $1,6 \mathrm{~mm}$ chez les plus grands individus: protopodite: 0,75 ; exopodite: 0.68 et endopodite: $0,68 \mathrm{~mm}$.

AFFINITÉS: Bragasellus escolai $\mathrm{n}$.sp., par ses divers caractéres, en particulier ceux des pléopodes I et II du mâle, se montre apparenté aux autres espéces déjà connues dans la moitié Nord de l'Espagne: B. lagari Henry et Magniez, 1973, des eaux souterraines des provinces de Cuenca, Guadalajara, Soria et Teruel et B.comasi Henry et Magniez, 1976, de la Cova del Infierno (Covadonga, Asturies). Il présente également de nettes affinités avec une espèce lusitanienne: B. seabrai (Braga, 1943) capturé dans des puits de Leça da Palmeira et Matozinhos, localités côtières situées immédiatement au NW de Porto: il s'agit d'une même lignée qui s'est diversifiée spécifiquement en colonisant les eaux souterraines du bassin du Douro.

Manuscrit terminé le 9 décembre 1978

RÉSUMÉ

Description d'une nouvelle espéce anophtalme et apigmentée de Bragasellus, collectée dans la grotte de Calderón, à Vellila de Carriòn, Province de Palencia. Espagne septentrionale )bassin hydrographique du Douro).

BIBLIOGRAPHIE

BRAGA J.M., 1943: Description de l'Asellus seabrai, isopode aveugle nouveau des eaux souterraines du Portugal. Mem. Est. Mus. Zool. Univ. Coimbra, 145, 12 pp.

HENRY J.P. et MAGNIEZ G., 1977; Les Asellides (Crustacea Isopoda Asellota) des eaux souterraines d'Espagne et leur répartition. Com. 6è. Simp. d'Espeologia, Terrassa (Barcelona), 1977: 29-36. 